\title{
A Platform Independent Architecture for Virtual Characters and Avatars *
}

\author{
M. Gillies ${ }^{1}$, V. Vinayagamoorthy ${ }^{1}$, D. Robeterson ${ }^{2}$, and A. Steed ${ }^{1}$ \\ ${ }^{1}$ Department of Computer Science, University College London, London, UK, \\ ${ }^{2}$ BT plc, Adastral Park, Ipswich IP5 3RE, UK \\ \{m.gillies, v.vinayagamoorthy, a.steed\}@cs.ucl.ac.uk, \\ dale.e2.robertson@bt.com
}

We have developed a Platform Independent Architecture for Virtual Characters and Avatars (PIAVCA), a character animation system that aims to be independent of any underlying graphics framework and so be easily portable. PIAVCA supports body animation based on a skeletal representation and facial animation based on morph targets.

An important features of PIAVCA is the "Motion Abstraction", a single abstract data type that represents any form of animation of a character. Different implementations of the abstraction can provide very different sources of animation, for example motion capture data, procedural animation or real time tracking data. The abstraction itself is very simple, each motion has a number of "tracks" each of which has a value that varies over time, which could be stored (in the case of motion capture data) but it could also be computed on the fly. It can represent both body animation and facial animation, where each track corresponds to a morph target. However, the real power of the motion abstraction is that is becomes possible to implement transformation on animations without knowing what the source of the animation is, or even without knowing whether it is a body or face animation. We have implemented a number of transformations on single motions (e.g. scaling, altering the speed, looping) and on multiple animations (e.g. smooth transitioning, interpolated motions, performing different motions on different parts of the body, randomly scheduling multiple motions). All these transformations are themselves implementations of the motion abstraction, making it possible to pass the result of one transformation to another one. This simple mechanism allows a new method of creating complex motions by composing multiple stages of transformations. The motion abstraction also makes PIAVCA highly extensible as new transformations or sources of animation merely have to implement the motion abstraction in order to be completely interoperable with existing ones.

PIAVCA also contains a number of other features that make it simple to use. It has a queue that makes it possible to smoothly sequence motions. It is also possible to play a number of "background motions" concurrently with other motions, for example, to provide variety and idling behaviour. PIAVCA has been used for a number of immersive virtual reality studies and we have developed a TCL-based framework for quickly creating experiemenal scenarios.

\footnotetext{
* This work has been supported by BT plc and European Union FET project PRES-
} ENCIA, IST-2001-37927 